\title{
Binding of organic ions to humic acids: spectroscopic and thermodynamic analyses of the ruling molecular interactions
}

Sedláček P. ${ }^{1}$, Smilek J. ${ }^{1}$, Kalina M. ${ }^{1}$, Enev V. ${ }^{1}$

${ }^{1}$ Institute of Physical and Applied Chemistry, Faculty of Chemistry, Brno University of Technology, Brno, Czech Republic, sedlacek-p@fch.vut.cz

doi: 10.36291/HIT.2019.sedlacek.045

Combination of aromatic or aliphatic structural moieties with pollar functional groups dissociable in an aqueous solution represents a structural motif abundant in anthropogenic environmental solutes such as pesticides, surfactants or antibiotics. The amphipathic structure provides these compounds with versatility of possible molecular interactions that they can be involved in. We have performed an extensive experimental study focused on binding of various model organic ions to humic substances of diverse origin. For this purpose, we have combined various analytical strategies, including spectroscopic assays (differential UV-VIS spectrometry, steady-state and time-resolved fluorescence spectroscopy), originally developed diffusion techniques (experimental evaluation of the solute molecular transport in a medium with homogenously dispersed humic substances), and examination of the binding thermodynamics based on (isothermal titration) calorimetry and equilibrium dialysis (see Fig. 1). This contribution evaluates the particular merits of the individual experimental techniques and summarizes the main findings on what type of molecular interactions plays a principal role in binding charged organic compounds by humic substances.

$$
t=0
$$

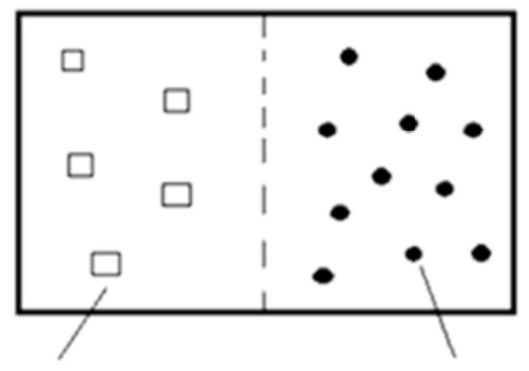

dispersed humics
(impermeant) charged solute (permeant) $t \rightarrow \infty$

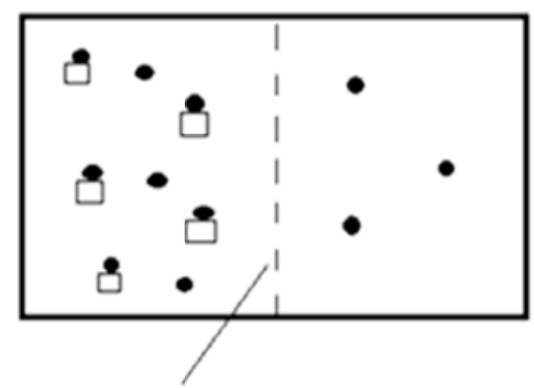

semipermeable membrane

Figure 1. Schematic diagram of the equilibrium dialysis experiment

Acknowledgements. This work was supported by Young Investigator Research Grant (Interaction of different (methylated) humic acids with organic ionic compounds) provided by International Humic Substances Society (IHSS). 